\title{
PLOTTER THOMPSON
}

Larissa Vanile Santos Carneiro1; Francimar Conceição dos Santos ${ }^{1}$; Michell Thompson Ferreira Santiago ${ }^{2}$; Rafael Maia Gadelha ${ }^{1}$; Tiago Jairo Vieira de Almeida ${ }^{1}$; Carlos Augusto F. de Jesus Ribeiro²; Tiara Borges Santiago Ferreira;

1 Faculdade Metropolitana de Camaçari - Tv. da Avenida Leste; Camaçari/BA; larissavanile@gmail.com

2 Universidade Federal da Bahia; R. Prof. Aristídes Novis, 2 - Federação, Salvador/BA;

${ }^{3}$ Universidade Cândido Mendes; Campos Rua Anita Peçanha, 100 - Pq. São Caetano - Campos dos Goytacazes/RJ

Resumo: A construção de um dispositivo plotter, controlado via Arduino similar a um CNC, baseou-se na necessidade de executar desenhos bidimensionais em superfícies sólidas, tendo como partes da sua estrutura física a utilização de tubos pvc e sucatas de equipamentos. Após a concretização da sua construção e do seu funcionamento, dando abertura para novas reformulações, com a possibilidade de inseri-la em aplicações destinadas ao campo da impressão, assim todos os objetivos foram alcançados, a construção e desenvolvimento do equipamento, análise e inserção de melhorias. Exaltando e reformulando o entendimento dos discentes ao que realmente seria a responsabilidade ambiental direcionando a percepção dos mesmos a novos horizontes relacionados ao descarte do lixo eletrônico.

Palavras-Chave: Plotter; Arduino; CNC; Tubos PVC.

\section{PLOTTER THOMPSON}

Abstract: The construction of a CNC-controlled by Arduino plotter device was based on the need to perform two-dimensional drawings on solid surfaces, using pvc duct and equipment scraps as part of its physical structure. After the completion of its construction and operation, opening up to new reformulations, with the possibility of inserting it in applications intended for the field of printing, thus all the objectives were achieved, the construction and development of equipment, analysis and insertion of improvements. Exalting and reformulating students' understanding of what environmental responsibility really would be, directing their perception to new horizons related to the disposal of electronic waste.

Keyswords: Plotter; Arduino; CNC; PVC duct. 


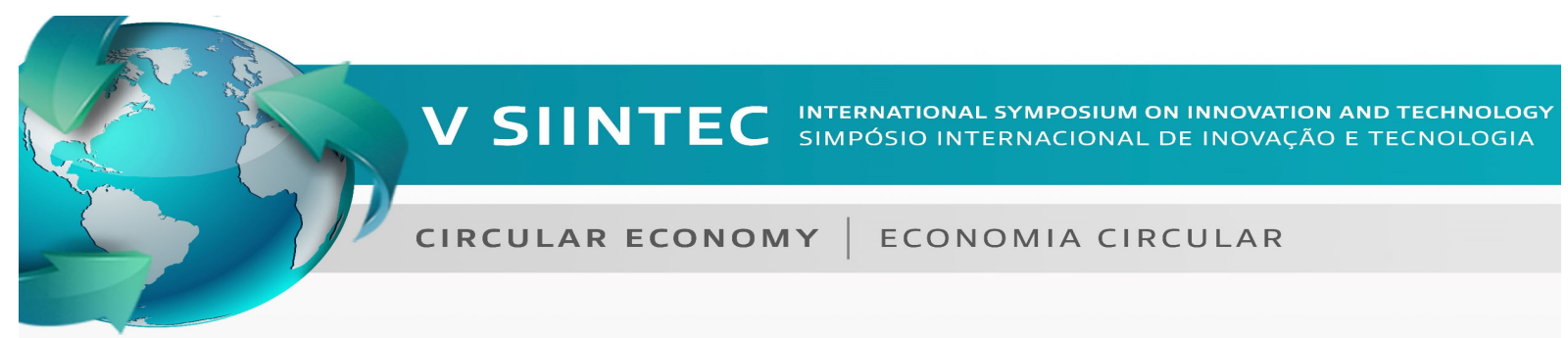

\section{INTRODUÇÃO}

O desenho a mão livre consiste na habilidade do indivíduo transpor em um papel uma determinada criação ou mesmo uma reprodução artística de um elemento físico ou abstrato, com o avanço dos processos de construção no decorrer dos séculos a aplicação do ato de desenhar em estruturas e monumentos tornou-se de extrema importância para tais fins; simplesmente passar para o papel deixou de ser apenas uma atividade manual, mas automatizada com uma extrema precisão de detalhes nas suas curvas e coloração, linhas extremamente retas fotos com alta qualidade e nitidez proporcionando uma melhor experiência de visualização.

Devido aos avanços tecnológicos o trabalho automatizado apresentou uma consistência nunca vista em qualquer trabalho manual suprindo as necessidades que um processo produtivo em grande escala necessita; demandando máquinas que consigam acompanhar o ritmo frenético que tais atividades se encontram suprindo essas novas necessidades.

Mesclando os desenvolvimentos anteriores chegou-se a constatação que impressões passariam a ir além do ato de tingir desbravaria novas superfícies além do papel, mas executar demarcações em equipamentos sólidos e até mesmo cortálos; dando origem ao nascimento de máquinas plotters que auxiliadas a softwares e ferramentas físicas possuíam a capacidade de executar este novo tipo de trabalho.

\section{METODOLOGIA}

O projeto originou-se na Faculdade Metropolitana de Camaçari (Famec), quando alguns membros da equipe que criaram a plotter, pensaram em desenvolver um equipamento capaz de demarcar placas de circuitos impresso profissionais, porém devido à dificuldade em desenhar linhas precisas sobre as placas utilizando a caneta de forma manual, o que era um grande problema, foi necessário a montagem de um router CNC (Computer Numeric Control) auxiliada com uma ferramenta de corte para desenhar as trilhas na placa de fenolite conforme a imagem abaixo:

Figura 1: Gravação em PCB com router CNC

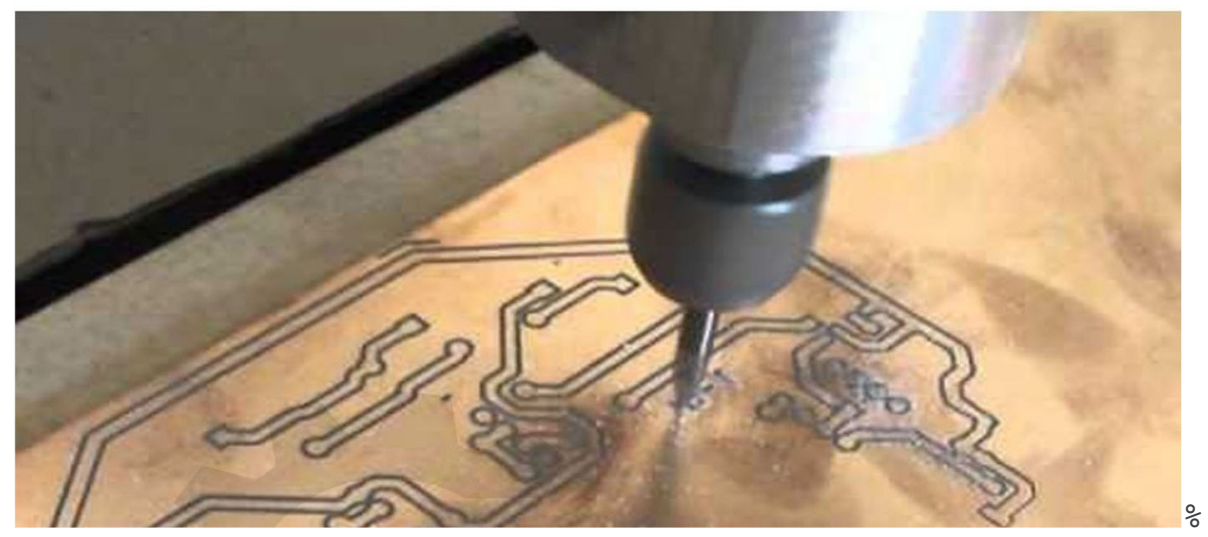


No processo de construção do corpo da máquina, foi utilizado sucatas para criar um equipamento que desenha no plano vetorial $\mathrm{x}, \mathrm{y}$. Tomando como base vetores de desenhos criados pelo computador, onde contém informações que o Gcode(Código $\mathrm{G}$ ) consegue converter para uma linguagem baseada em códigos que são interpretados pela biblioteca GRBL (firmware open source) e executa como coordenadas, modelando o mesmo com motores de passo e correias dentadas. Foi utilizado tubos PVC (Polyvinyl chloride) como guias, um marcador permanente como ferramenta de desenho e um servo motor como solenoide, que faz o controle do marcador.

A precisão do desenho foi melhorado ao longo do projeto, onde foi possível obter os resultados esperados, todavia para dar início ao projeto, verificou-se que teria um custo elevado para a sua construção, por conta da imprescindibilidade em comprar ferramentas de corte e outros equipamentos, então para reduzir a despesa, foi decidido montar uma plotter $2 \mathrm{D}$, que segue o mesmo princípio das que são construídas para cortar, no entanto a ferramenta de corte, seria substituída por um marcador permanente, que desenha as trilhas de forma precisa na placa de fenolite para fazer a corrosão do cobre com o percloreto de ferro.

Onde o dispositivo é controlado por eixos e motores que recebem coordenadas de um microcontrolador Arduino uno, que atua em suas saídas para ativar os motores de acordo com o que o GRBL manda de sinal, ou seja, a sequência seguida para acionamento deles é feita pelo arduino de comandos recebidos pelo GRBL. Neste projeto foi reutilizado materiais que seriam descartados, entre eles: tubos e conexões pvc, pedaço de chapa de madeira, correias de máquina Singer, fonte de alimentação de vídeo game e engrenagens de ferro. Porém houve a necessidade de adquirir alguns componentes como: Arduino, cnc shield v3, Motores de passo, Servo Motor, fita Hellerman e parafusos.

\subsection{Descrições dos Componentes}

\subsubsection{Arduino UNO R3}

Um Arduino é um pequeno computador que você pode programar para processar entradas e saídas entre o dispositivo e os componentes externos conectados a ele. O Arduino é que chamamos de plataforma de computação física ou embarcada, ou seja, um sistema que pode interagir com seu ambiente por meio de hardware e software [1].

\subsubsection{Cnc Shield v3}

O Cnc Shield v3 é uma placa que faz a conexão dos drivers de potência, fonte de energia e demais periféricos com o Arduino UNO. Este shield simplifica a instalação eletrônica e possui encaixes para o acoplamento direto de até quatro drivers de potência, com circuito de alimentação de energia tanto para o Arduino quanto para os drivers [2]. 


\subsubsection{Motor de Passo}

Segundo Brites e Santos [3], os Motores de Passo são dispositivos eletromecânicos que convertem pulsos elétricos em movimentos mecânicos que geram variações angulares discretas. $O$ rotor ou eixo de um motor de passo e rotacionado em pequenos incrementos angulares, denominados "passos", quando pulsos elétricos são aplicados em uma determinada sequência nos terminais deste. A rotação de tais motores é diretamente relacionada aos impulsos elétricos que são recebidos, bem como a sequência a qual tais pulsos são aplicados reflete diretamente na direção a qual o motor gira. A velocidade que o rotor gira é dada pela frequência de pulsos recebidos e o tamanho do ângulo rotacionado é diretamente relacionado com o número de pulsos aplicados."

\subsubsection{Servo Motor}

Conforme Neto [4], o servo motor é um dispositivo eletromecânico que, a partir de um sinal elétrico em sua entrada, pode ter seu eixo posicionado em uma determinada posição angular. Servo motores não foram feitos para girar livremente, e sim para ir para uma posição escolhida de acordo com um limite, que é geralmente é de 180 graus. Por serem pequenos, compactos, além de permitir um posicionamento preciso de seu eixo, eles são comumente utilizados em robótica e modelismo.

\subsubsection{GRBL}

O Grbl (pronuncia-se "gerbil") é um firmware desenvolvido e otimizado para ser gravado em microcontroladores atmega328p (sendo estes presentes em placas Arduino UNO compatíveis), com a finalidade de controlar os movimentos de máquinas operatrizes. Trata-se de um software livre, lançado sob a licença GPLv3 e desenvolvido em $\mathrm{C}$, que requer um hardware simples e opera seguindo os padrões industriais. Por estes motivos, o Grbl se tornou um fenômeno entre as plataformas de código aberto, sendo base para máquinas $\mathrm{CNC}$, impressoras 3D entre outros [2].

\subsubsection{G-code}

G-code é o nome genérico para a linguagem de programação utilizada para controle de máquinas-ferramentas. Quando um desenho é feito no computador, ele contém informações que devem ser convertidas para uma linguagem que a máquina irá entender e o Gcode é a linguagem responsável por essa interpretação. Quando o usuário aciona um botão para movimentar uma máquina CNC ou quando carrega um arquivo para iniciar a criação de uma peça, linhas de comandos em G-code são 
enviados para a máquina onde um controlador as interpreta e transforma os comandos em movimentos ou aciona ferramentas [2].

\subsubsection{Correias e Polias}

Dentro do par correia-polia, existe uma variedade de tipos de transmissões, por correias planas, em $\mathrm{V}$ e correias dentadas ou sincronizadoras podendo todas estas serem acompanhadas de polias das mesmas variedades sendo a correia plana a mais comum dos tipos.

O par correia-polia e uma alternativa barata para transmissões mecânicas, já que os elementos não precisam de grande precisão para atingir grandes rendimentos, sendo geralmente superiores a $90 \%$.

Quando instalando uma correia, uma preocupação deve ser a tensão mecânica. A tensão não pode ser muito alta, pois pode causar muito atrito na correia ou muito esforço no eixo, mas se a tensão for muito baixa a transmissão entre eixos terá um menor rendimento.

A principal vantagem das correias dentadas e que os dentes delas se encaixam nos dentes da roda dentada acoplada no eixo motriz, o que a faz agir quase como uma corrente e diminui um dos maiores problemas das correias, o escorregamento. [2]

\subsection{Listas de Materiais}

4 Cano PVC $20 \mathrm{~mm}$ com 3,5 cm de comprimento.

2 Cano PVC $20 \mathrm{~mm}$ com 5,5 cm de comprimento.

4 Cano PVC $20 \mathrm{~mm}$ com 9,5 cm de comprimento.

4 Cano PVC $20 \mathrm{~mm}$ com $17,5 \mathrm{~cm}$ de comprimento.

2 Cano PVC $20 \mathrm{~mm}$ com $40 \mathrm{~cm}$ de comprimento.

4 Cano PVC $20 \mathrm{~mm}$ com $30 \mathrm{~cm}$ de comprimento.

8 Cano PVC $25 \mathrm{~mm}$ com $3 \mathrm{~cm}$ de comprimento.

6 Joelhos $90^{\circ}$ de cano $20 \mathrm{~mm}$

8 Ts $90^{\circ}$ de cano de $20 \mathrm{~mm}$

1 Arduino uno

1 CNC Shield V3

4 Drivers A4988

2 Motor de passo nema 17 ZEPHYR

1 Servo motor $9 g$ 
1 fonte de alimentação

4 Engrenagens de $20 \times 20$ de passo 4

2 Correias dentadas de passo 4

1 Chapa de madeira $200 \mathrm{~mm} \times 200 \mathrm{~mm}$

10 fitas hellerman

4 Prendedores de papel

\section{RESULTADOS E DISCUSSÃO}

Ao longo do projeto, foi constatado que a plotter estava sem estabilidade no marcador, evidenciando a necessidade de fazer algumas melhorias com a impressora 3D, sendo eles um suporte para segurar e fixar tanto o marcador quanto o servo motor, também foram feitas algumas engrenagens, para que todas ficassem padronizadas, com a intenção de movimentarem-se em sincronia.

Foram projetados alguns suportes para estabilizar os motores de passo, na estrutura, a fim de que conseguissem manter uma maior aderência e estabilidade, deste modo foi possível oferecer mais qualidade e precisão para os desenhos, conservando a ideia de um bom custo benefício.

Durante o processo foi constatado que na utilização do marcador (caneta de tinta comum), ocorria o deslocamento do seu módulo de elasticidade na sua estrutura física, devido a pressão exercida pelos eixos, que era causado pelo atrito imposto pela placa, com isso provocando a diminuição da precisão ao fazer curvas e as demarcações dos círculos onde seriam feitas as perfurações para inserir os componentes.

Optou-se em substituir o suporte do marcador por um laser de drive de DVD (Digital Versatile Disc), ao utilizar o diodo laser removido do drive foi observado que a luz emitida por ele, não estava concentrada em um único ponto, sendo insuficiente para fazer demarcações nas placas, devido a dispersão da luz em uma área maior. Não houve um efeito satisfatório, perdendo a sua eficiência.

Foi realizado no projeto a inserção da lente presente no drive, tendo por função direcionar toda a luz nas áreas desejadas sendo capaz de executar com exatidão as gravações na placa de fenolite. 


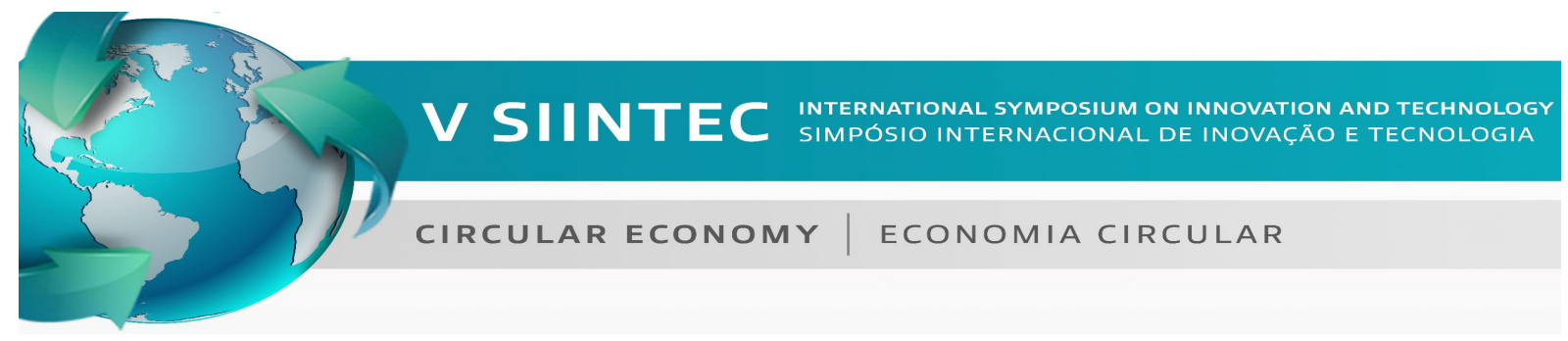

Figura 2: Frame Plotter

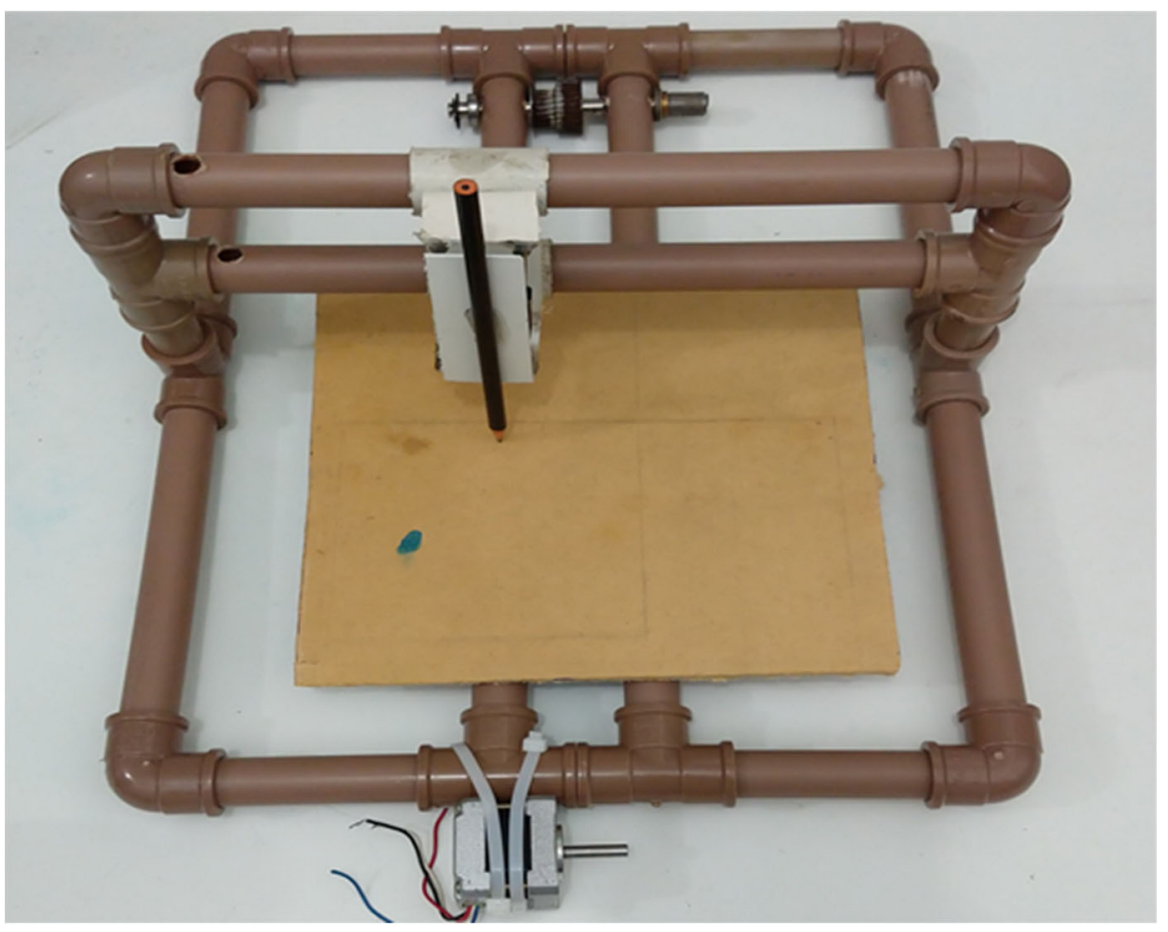

Figura 3: Plotter executando um desenho.

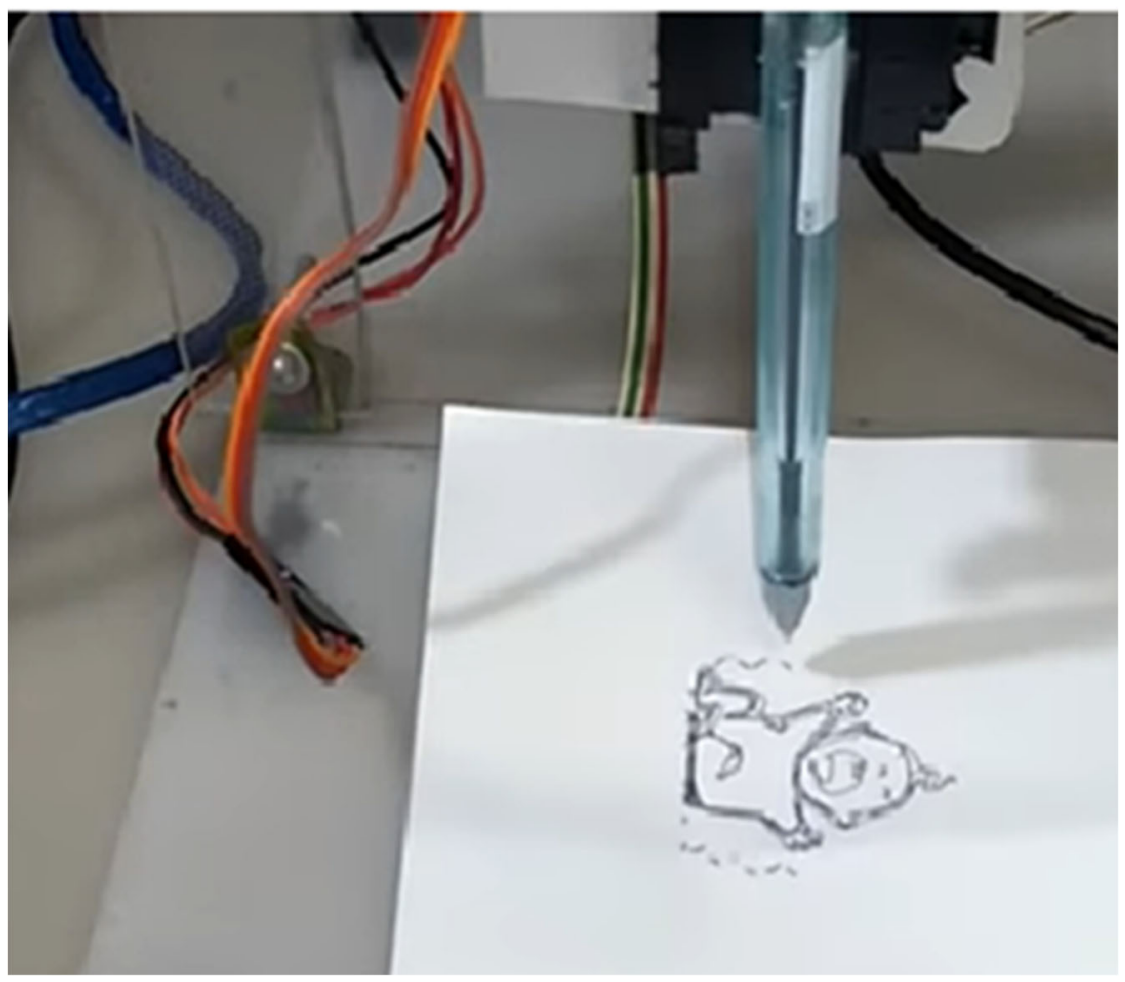




\section{CONCLUSÃO}

Iniciando novas formas de aprimorar os conhecimentos além dos livros didáticos, pensou-se no desenvolvimento e implantação de experimentações práticas baseadas em projetos já existentes, compartilhando com os discentes as principais diretrizes básicas para o seu desenvolvimento criativo, por meio do manuseio de ferramentas, montagem e desmontagem de componentes eletrônicos. Além da concepção da linguagem de programação, garantindo autonomia para que os alunos construam suas próprias plotters.

Promovendo a proposta de sustentabilidade, foi realizado o reaproveitamento de peças e materiais de sucata para a composição do plotter, e como programação dos comandos, foi utilizada a plataforma de prototipagem eletrônica de hardware livre Arduino.

\section{REFERÊNCIAS}

[1] THOMPSON, M. Arduino Básico. São Paulo. NOVATEC Editora, 2011.

[2] POLASTRINI, F. H. Desenvolvimento de uma Máquina CNC de Baixo Custo com Software e Hardware Abertos. 2016. 100f. Bacharel em Engenharia Elétrica. Instituto Federal Minas Gerais, Formiga.

[3] BRITES, F. G.; SANTOS, V. P. de A. Motor de Passo. 2008. 15f. Bacharel em Engenharia de Telecomunicações. Universidade Federal Fluminense. Niterói.

[4] MARQUES NETO, M. C. Desenvolvimento de Aplicações Ubíquas com Arduino e Raspbery Pi. 2014. 30f. Instituto Federal de Educação, Ciência e Tecnologia da Bahia. Salvador. 